\title{
Nanopatterning Au chips for SPR refractometer by using interference lithography and chalcogenide photoresist
}

\author{
V.A. Dan'ko, G.V. Dorozinsky, I.Z. Indutnyi, V.I. Myn'ko, Yu.V. Ushenin, P.E. Shepeliavyi, \\ M.V. Lukaniuk, A.A. Korchovyi, R.V. Khrystosenko \\ V. Lashkaryov Institute of Semiconductor Physics, NAS of Ukraine \\ 41, prospect Nauky, 03680 Kyiv, Ukraine \\ E-mail: indutnyy@isp.kiev.ua
}

\begin{abstract}
This study reports on development of the interference lithography (IL) technique applying the resist based on chalcogenide glass films for fabrication of gold chips in the form of periodic surface nanostructures for surface plasmon resonance (SPR) refractometers. The IL technique was optimized for patterning the Au layers and formation of one-dimensional (grating) structures with the spatial frequency close to $3300 \mathrm{~mm}^{-1}$. The spatial frequency and depth of grating grooves were selected with account of the condition for Bragg reflection of plasmons at the operation wavelength of SPR refractometer and given environment (which is a condition for enhancing biosensor sensitivity as compared to that of a flat Au chip surface). It has experimentally been demonstrated that the use of diffraction patterns in SPR sensor increases its response by $17 \%$.
\end{abstract}

Keywords: interference lithography, surface plasmon resonance, chalcogenide glass films, photoresist.

Manuscript received 12.05.15; revised version received 18.08.15; accepted for publication 28.10.15; published online 03.12.15.

\section{Introduction}

Surface plasmon resonance (SPR) devices have found wide use in recent years in biosensing applications due to their advantages of high sensitivity, label-free, realtime and rapid detection. During the last two decades, it has been made a great progress in the development of instrumentation and practical applications [1,2].

But conventional reflection-type SPR biosensors employing a prism in the attenuated total reflection configuration (Kretschmann configuration) [3] present several drawbacks and usually lack the sensitivity to detect interaction of proteins with small ligands [4]. To improve the performance of SPR sensors, sensitivity enhancement has been of tremendous interest in the development of these sensors. In recent years, modified SPR sensing systems, such as gold nanoparticles immobilized on a gold film [5], gold nanoparticleembedded dielectric layer on a gold film [6], and metallic nanowires regularly patterned on a metal film [7], have been proposed theoretically and experimentally. Enhanced sensing characteristics are mainly associated with shift or bending in dispersion relation of propagating surface plasmon, induced by its strong coupling with localized plasmon resonance modes in metallic nanostructures [8].

The recent theoretical study [9] has shown that by formation a sinusoidal profile grating at the surface of the metal layer of the SPR sensor used in the Kretschmann configuration, the biosensor sensitivity 
may be enhanced in comparison with that of uncorrugated metal surface. Surface plasmons propagating in the direction perpendicular to a one-dimensional grating (i.e. along the grating vector) will experience back reflections. If the period of the grating corresponds to the Bragg condition, then a bandgap will form where no surface plasmons are permitted to propagate. The control over the position of the bandgap with respect to the wavelength and angle can be accomplished through the manipulation of the period and/or grating height. By designing the operating point of the sensor to be near the bandgap, the sensitivity can be improved as compared to the sensitivity with flat metallic layer. But these results have not yet been tested experimentally.

In order to get good plasmon resonance and enhanced sensor performance, high-quality metallic gratings or another periodic submicrometer structures over large areas are needed. With an advance of nanofabrication technology, periodic metallic nanostructures such as nanodot, nanohole and nanograting have been realized in order to acquire designable and predictable plasmonic properties. Depending on the period and total surface area of these structures and the materials to be used, a number of different fabrication techniques can be considered. Last decade, interference (interferometric) lithography (IL) is widely used for fabrication of defect-free periodic microand nanostructures. IL is a large area fabrication technique that uses laser interference patterns for rapid formation of periodic structures such as gratings, and bigratings (arrays). The total processed area depends on the beam intensity and coherence length of the laser and can be up to dozens, or even hundreds of square centimeters. The technology is much cheaper and simpler than the electron beam lithography and can be used for manufacturing of SPR sensor structures $[10,11]$.

In previous studies, the authors have shown that IL with the use of chalcogenide photoresist is a promising technology to form one- and two-dimensional submicron periodic structures on the surface of semiconductors and dielectrics [12]. Chalcogenide photoresists based on thermal evaporated amorphous films of chalcogenide glasses are characterized by high resolution, optical uniformity, wide spectral range of photosensitivity. In addition, these photoresists possess a high refractive index ranging from 2.0 up to 3.0 and are very perspective for immersion IL.

In this paper, we report the IL technique with the use of resist based on the chalcogenide glass $(\mathrm{ChG})$ films for fabrication of gold chips with periodic surface nanostructures for SPR refractometers.

\section{Experimental}

The samples for our experiments were prepared by successive thermal vacuum deposition of $1 \ldots 3 \mathrm{~nm}$ thick (effective thickness) $\mathrm{Cr}$ adhesive layer, a layer of metal $\mathrm{Au}$ ) with the thickness of 40 to $50 \mathrm{~nm}$ and photoresist layer $\left(\mathrm{As}_{2} \mathrm{~S}_{3}\right.$ or $\left.\mathrm{GeSe}_{3}\right)$ with the thickness from the range 50 to $300 \mathrm{~nm}$ onto polished glass substrates (F-1 glass,
$20 \times 20 \mathrm{~mm}$ ) at the residual pressure of $2 \cdot 10^{-3} \mathrm{~Pa}$. The deposition rate and film thickness were monitored in situ with a KIT-1 quartz thickness meter. After deposition, the film thickness was controlled using a MII-4 microinterferometer. To increase stability of the layer, the gold chips were annealed at $T \sim 120^{\circ} \mathrm{C}$ before chalcogenide deposition [13].

Recording the periodic structures on $\mathrm{ChG}$ films was carried out using the interference pattern formed with a helium-cadmium laser (wavelength $\lambda=440 \mathrm{~nm}$ ). The exposition value for the gratings recording (1D structures) was $0.2 \ldots 0.5 \mathrm{~J} / \mathrm{cm}^{2}$. After exposure, the samples were chemically treated in non-water alkaline organic solutions to form a resistive mask in the photoresist layer, through which the metal film was etched. After removing the photoresist residues in alkaline solution, washing and drying the metal periodic structure was obtained.

The surface patterns of obtained structures were examined with a Dimension 3000 Scanning Probe atomic force microscope (Digital Instruments Inc., Tonawanda, NY, USA) in the AFM tapping mode.

Measurements of angular dependences for light reflection (reflection curve) from the interface analyt/gold film on glass substrate were performed using the two SPR refractometers Plasmon-6 and Plasmon-71 (V. Lashkaryov Institute of Semiconductor Physics, NAS of Ukraine, Kyiv) with the operation wavelengths 650 and $850 \mathrm{~nm}$, respectively. These devices allow measuring the absolute value of reflectivity as well as absolute value of the angle in the Kretschmann configuration. The wide range of angles for scanning (up to 17 degree in air) allows performing quantitative measurements of a full reflection curve for liquids with various refraction indexes - from 1.33 (water) up to 1.41 (hemoglobin) for Plasmon-6 and from 1.33 (water) up to 1.47 (motor oil) for Plasmon-71 [14, 15].

\section{Results and discussion}

Theoretical modeling in [9] was carried out for a sinusoidal surface relief grating with a small depth of the relief. For these gratings, we can approximately estimate the value of the Bragg period $\left(\Lambda_{B}\right)$ using the following simple formula:

$\Lambda_{\mathrm{B}}=0.5 \lambda_{0}\left[\left(\varepsilon_{m r}+\varepsilon_{D}\right) / \varepsilon_{m r} \varepsilon_{D}\right]^{1 / 2}$,

where $\lambda_{0}$ is the free-space wavelength, $\varepsilon_{D}$ - dielectric constant of the dielectric and $\varepsilon_{m r}$ - real part of the dielectric constant inherent to the metal.

It was obtained that, for our samples (Au layer on F-1 glass substrates) at the wavelength $650 \mathrm{mn}$, the conditions of Bragg reflection of plasmons (which is the condition for enhancing the biosensor sensitivity as compared to that for a flat surface) are satisfied at spatial frequencies of gratings near $3000 \mathrm{~mm}^{-1}$ for the interface $\mathrm{Au}$-air. For the interface Au-water, this condition is satisfied for the same spatial frequency at the operation wavelength $850 \mathrm{~nm}$. 
The IL technique was optimized (thickness of photoresist, exposure time, selectivity of etchant and time of the post-exposure etching) for patterning the $\mathrm{Au}$ layer and formation of one-dimensional structures with the spatial frequency up to $3300 \mathrm{~mm}^{-1}$. IL technology was applied in the mode of little over-exposure of photoresist to provide a cycloid form of groove profile of periodic chalcogenide mask. By changing the time of selective etching of photoresist, it is possible to change the width of the elements of lithographic masks and, accordingly, the width of opened intervals between the elements of the mask, through which further etching the metal layer is fulfilled. Etching the photoresist was monitored in situ by registration of non-photoactive longwave light diffracted from relief structure that is formed in the photoresist layer. For a given photoresistive mask, a form of profile and duty cycle (the ratio of width to period) of the elements in the periodic structure, obtained as a result of wet etching the metal layer through the chalcogenide mask, were also determined by the rate and time of metal etching.

Fig. 1a shows the AFM image of the gold grating formed using IL on the gold layer with a thickness near $40 \mathrm{~nm}$ by wet etching through $\mathrm{ChG}$ resistive mask. The period of this grating is $330 \mathrm{~nm}$ (spatial frequency $3000 \mathrm{~mm}^{-1}$ ). On this sample, the photoresist mask was formed with the ratio of the strip width to interval width close to unity. The depth of the relief reaches $36 \mathrm{~nm}$, and the groove sectional profile is trapezoid, even close to rectangular with flat tops (Fig. 1b). It means that the gold layer is etched throughout the whole thickness to the glass substrate.

This grating was annealed in vacuum at $400{ }^{\circ} \mathrm{C}$ for $15 \mathrm{~min}$. Fig. 2 shows the image and groove profile of the same grating after annealing. One can see significant changes in the groove profile as a result of annealing. Trapezoid flat-topped groves were transformed to convex grooves with a smaller half-width and greater height. Investigation of groove heights histogram has shown that the initial height of the grooves of $36 \pm 2 \mathrm{~nm}$ has increased up to $49 \pm 5 \mathrm{~nm}$ as a result of the annealing. That is the flat gold nano-strips were transformed during the annealing into semi-cylindrical nano-wires by the forces of surface tension.

So, using the additional annealing we can change the shape of the grating grooves, and to some extent vary condition (resonance angle, wavelength) of a plasmon resonance.

Enhancement of the biosensor sensitivity was theoretically studied [9] for a grating on the Ag surface with the depths of the relief 20 and $40 \mathrm{~nm}$ (respective total thicknesses of the metal layers were 70 and $90 \mathrm{~nm}$ ). The optimal thickness of gold films for SPR refractometer chips is $45 \ldots 50 \mathrm{~nm}$. To investigate optical characteristics and sensitivity of the sensors with diffraction grating, we produced several gratings with a small depth of relief and the spatial frequency close to $3000 \mathrm{~mm}^{-1}$ on the gold film with the thickness $50 \mathrm{~nm}$.

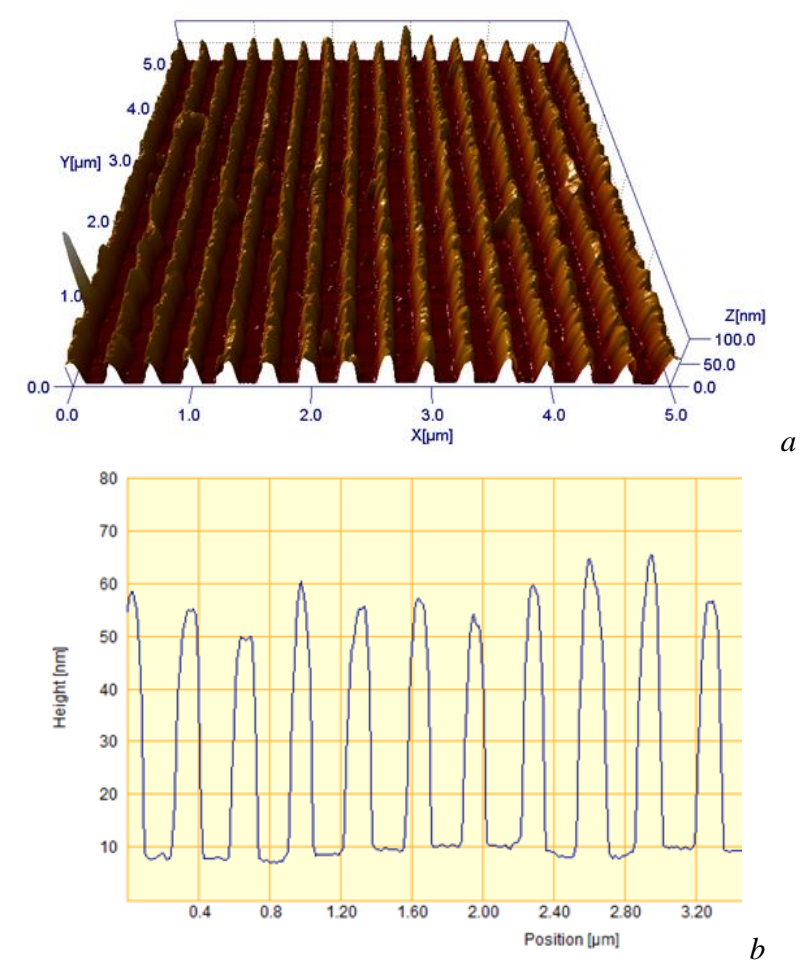

Fig. 1. AFM image and cross-sectional profile of the Au grating with the period $330 \mathrm{~nm}$.
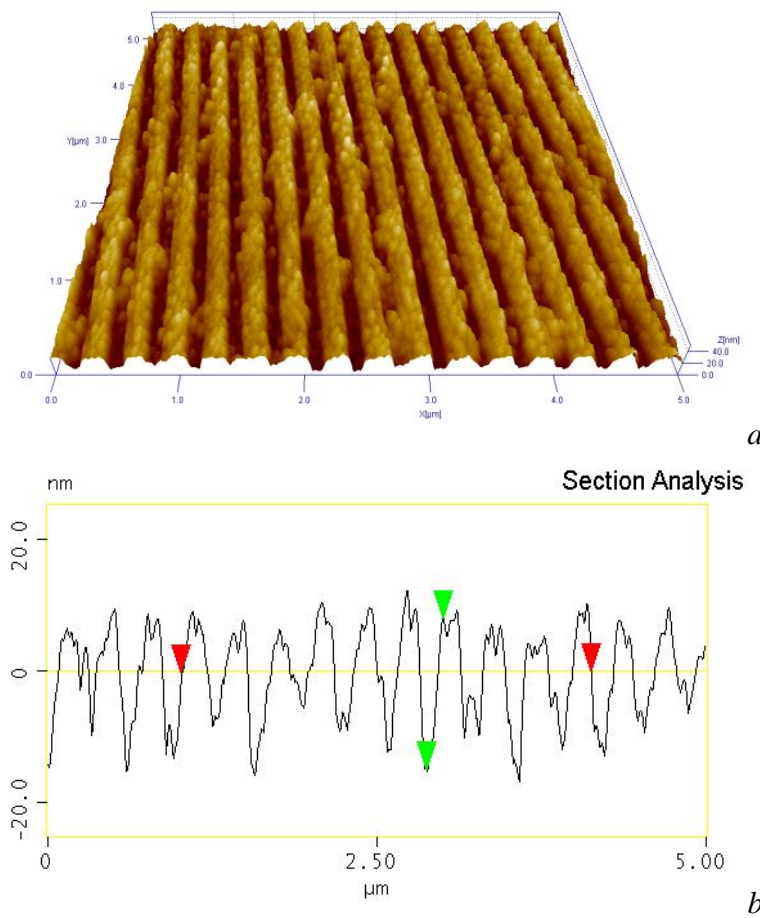

Fig. 2. The same grating as in Fig. 1 after annealing for $15 \mathrm{~min}$ at $400{ }^{\circ} \mathrm{C}$.

Fig. 3 shows the AFM image and cross-sectional profile of the Au grating with the period $330 \mathrm{~nm}$ and relief depth close to $20 \pm 4 \mathrm{~nm}$. 

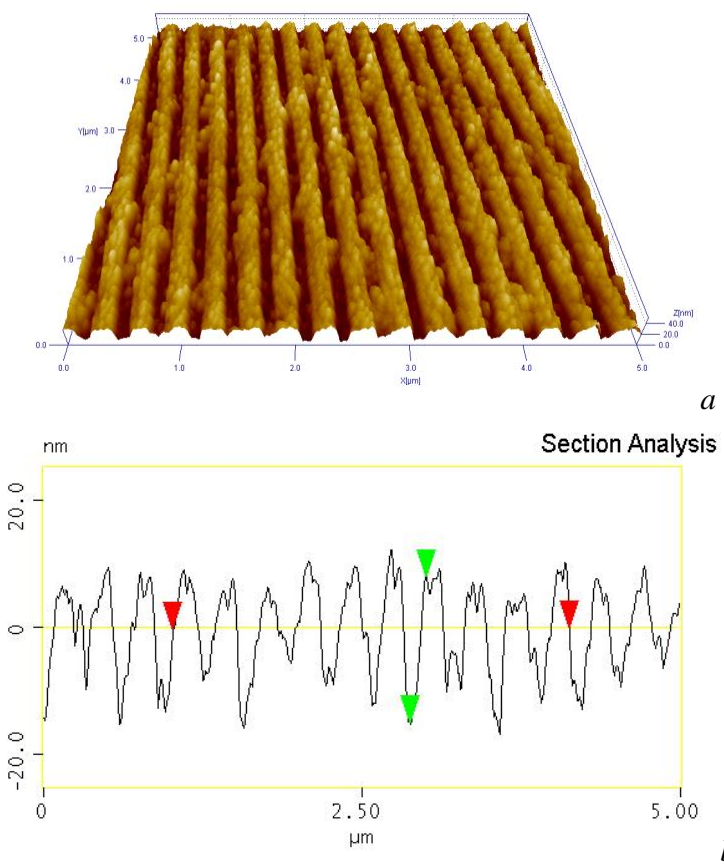

Fig. 3. AFM image and profile of the Au grating with the period $330 \mathrm{~nm}$ and depth of the relief $20 \pm 4 \mathrm{~nm}$ (total thickness of Au layer is $50 \mathrm{~nm}$ ).

Formation of diffraction grating on the surface of the gold film changes the shape of the angular dependence for reflection from the operation surface of the sensor near resonance. Fig. 4 shows the curves of surface plasmon resonance for a standard sensor with the flat surface of gold film (black line) and sensor with a diffraction grating (white line) with the same parameters (spatial frequency, depth relief) as for the grating in Fig. 3. The external environment in these measurements was air. The dependence of the reflectance on the angle of incidence (SPR curves) were measured using the device "Plasmon-6" (operation wavelength $-650 \mathrm{~nm}$ ). From the figure, one can see that SPR curve for the sensor with grating is significantly expanded and shifted to greater angles. In addition, two maxima are seen, indicating proximity to the Bragg resonance.

For comparison, study of sensor sensitivity was carried out for both sensors in liquid environment. SPR curves were measured using the device "Plasmon-71" (operation wavelength $-850 \mathrm{~nm}$ ). Fig. 5 shows the SPR curves for the sensor with a diffraction grating possessing the same parameters as in Fig. 3, and Fig. 6 shows the SPR curves for a standard sensor.

The external environments in these measurements were distilled water (white lines on both graphs) and 96\% aqueous solutions of ethanol (black lines). Their refraction indexes were measured independently using the Abbé refractometer IRF-22 and were 1.3272 for water and 1.3584 for ethanol solutions. The angular position of SPR minimum was calculated for two ambient liquids. Also, we calculated enhancement of the sensor response $(S)$ with a diffraction grating as compared to the sensor without it. $S$ was calculated as the ratio $\Delta \theta_{d s p} / \Delta \theta_{s p}$, (where $\Delta \theta_{d s p}$ is the shift of angular position for SPR minimum for the sensor with grating at the transfer from water to ethanol, $\Delta \theta_{s p}$ - the same value for the standard sensor) and was 1.17 .

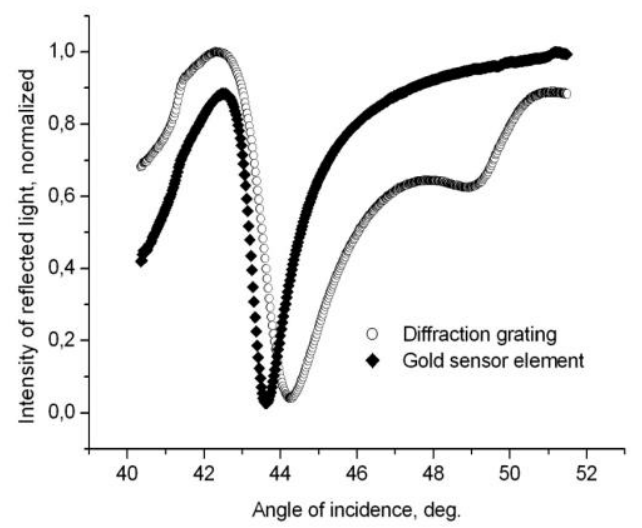

Fig. 4. SPR curves plotted for a sensing element with a diffraction grating (white line) and without it (black line) in ambient air. SPR curves were measured using the device "Plasmon-6".

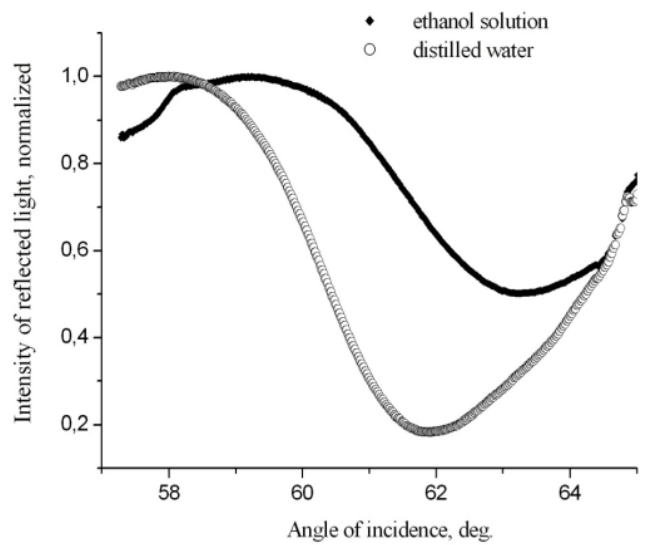

Fig. 5. SPR curves for distilled water (white line) and ethanol solution (black line) for sensing element with diffraction grating. SPR curves were measured using the device "Plasmon-71".

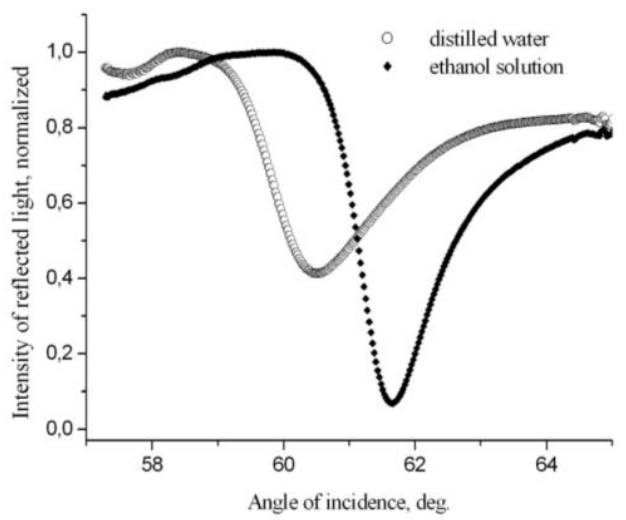

Fig. 6. SPR curves for distilled water (white line) and ethanol solution (black line) for standard sensor. SPR curves were measured using the device "Plasmon-71". 
Thus, the use of diffraction patterns in SPR sensor increases sensor response by $17 \%$. This value is much higher than the possible experimental error (experimental accuracy for determination of the angular position of SPR minimum for "Plasmon-71" is 0.006 degrees, and in our experiment $\Delta \theta_{s p}=1.147^{\circ}$ and $\Delta \theta_{d s p}=$ $\left.1.338^{\circ}\right)$, but is smaller than the theoretically possible [9] maximum value $(600 \%)$. It should be noted that enhancement of sensitivity, according to the simulation results [9], strongly depends on the refractive index of the environment on the external surface of the gold film. Significant enhancement should be observed in a narrow range of variation of the refractive index $(\Delta n \leq 0.01)$ for given grating parameters. A large interval of $\Delta n$ (as in our case $\Delta n \sim 0.03$ ) gives the average value of enhancement, which should be much less than the theoretically possible maximum. In addition, theoretical modeling were performed for the ideal structures without surface roughness and other possible defects. Such inhomogeneities can cause scattering of plasmons, broadening of the resonance curve and decrease the sensor sensitivity. To obtain higher sensitivity, further optimization in fabrication procedure and parameters of gratings is currently underway.

\section{Conclusion}

Being based on the presented results, we can conclude that IL method with additional annealing enables successful fabrication of patterned SPR chips with desired characteristics (spatial frequency, depth of relief, form of profile) on the area larger than $5 \mathrm{~cm}^{2}$.

The results of measuring the sensor sensitivity in liquid environment confirm theoretical prediction about enhancement of SPR biosensor sensitivity by formation of a grating with the appropriate period and depth on the rear face of SPR chip.

The authors gratefully acknowledge that this work have been partly funded by the Swiss National Science Foundation (SNSF, Bern) under grant no. IZ73Z0_152661 (SCOPES).

\section{References}

1. J. Homola, Surface plasmon resonance sensors for detection of chemical and biological species // Chem. Rev. 108(2), p. $462-493$ (2008).

2. B.A. Snopok, E.V. Kostyukevich, S.I. Lysenko et al., Optical biosensors based on the surface plasmon resonance phenomenon: optimization of the metal layer parameters // Semiconductor Physics, Quantum Electronics and Optoelectronics, 4(1), p. 56-69 (2001).

3. E. Kretschmann, H. Raether, Radiative decay of non-radiative surface plasmons excited by light // Z. Naturforsch. A, 23, p. $2135-2136$ (1968).
4. P. Englebienne, A. Van Hoonacker, M. Verhas, Surface plasmon resonance: principles, methods and applications in biomedical sciences // Spectroscopy, 17(2, 3), p. 255-273 (2003).

5. R.S. Moirangthem, Y.-C. Chang, and P.-K. Wei, Ellipsometry study on gold-nanoparticle-coated gold thin film for biosensing application // Biomed. Opt. Exp. 2(9), p. 2569-2576 (2011).

6. W.P. Hu, S.-J. Chen, K.-T. Huang, J.H. Hsu, W.Y. Chen, G.L. Chang, and K.-A. Lai, A novel ultrahigh resolution surface plasmon resonance biosensor with an $\mathrm{Au}$ nanocluster-embedded dielectric film // Biosens. Bioelectron. 19(11), p. 1465-1471 (2004).

7. K.M. Byun, S.J. Yoon, D. Kim, and S.J. Kim // Experimental study of sensitivity enhancement in surface plasmon resonance biosensors by use of periodic metallic nanowires // Opt. Lett. 32(13), 1902-1904 (2007).

8. Nak-hyeon Kim, Munsik Choi, Jung Woo Leem et al., Improved biomolecular detection based on a plasmonic nanoporous gold film fabricated by oblique angle deposition // Opt. Exp. 23(14), p. $18777-18785$ (2015).

9. C.J. Alleyne, A.G. Kirk, R.C. McPhedran, N-A.P. Nicorovici and D. Maystre // Enhanced SPR sensitivity using periodic metallic structures // Opt. Exp. 15, p. 8163-8169 (2007).

10. A. Arriola, A. Rodriguez, N. Perez et al., Fabrication of high quality sub-micron Au gratings over large areas with pulsed laser interference lithography for SPR sensors // Opt. Mater. Exp. 2(11), p. 1571-1579 (2012).

11. M. Vala and J. Homola, Flexible method based on four-beam interference lithography for fabrication of large areas of perfectly periodic plasmonic arrays // Opt. Exp. 22(15), p. 18778 (2014).

12. V. Dan'ko, I. Indutnyi, M. Min'ko, P. Shepelyavyi, Interference photolithography with the use of resists on the basis of chalcogenide glassy semiconductors // Optoelectronics, Instrumentation and Data Processing, 46(5), p. 483-490 (2010).

13. B.A. Snopok, E.V. Kostyukevich, S.I. Lysenko et al., Optical biosensors based on the surface plasmon resonance phenomenon: optimization of the metal layer parameters // Semiconductor Physics, Quantum Electronics and Optoelectronics, 4(1), p. 56-69 (2001).

14. N. Gridina, G. Dorozinsky, R. Khristosenko, V. Maslov, A. Samoylov, Yu. Ushenin, Yu. Shirshov, Surface plasmon resonance biosensor // Sensors \& Transducers J. 149(2), p. 60-68 (2013).

15. G.V. Dorozinsky, A.I. Liptuga, V.I. Gordienko, V.P. Maslov, V.V. Pidgornyi, Diagnostics of motor oil quality by using the device based on surface plasmon resonance phenomenon // Scholars J. Eng. and Technol. (SJET), 3, p. 372-374 (2015). 